\title{
Magnetic Resonance Imaging (MRI) in Syringomyelia
}

\author{
H. L. J. Tanghe \\ Department of Radiodiagnostics, University Hospital Dijkzigt, Rotterdam, The Netherlands
}

\section{Summary}

Based on an own material of 19 patients with syringomyelia and on the related literature a survey is given on the diagnosis, differential diagnosis, postoperative evaluation and the dynamics of CSF and cyst fluids, using magnetic resonance imaging (MRI).

The following conclusions can be drawn:

1. MRI is the preferred method of investigation for diagnosis and differential diagnosis of syringomyelia.

2. Using MRI, it is possible to study fluid flow in the vertebral canal and the movements of the syrinx fluid. The data are as yet limited, but in the future will form an important contribution to our understanding of the pathogenesis of syringomyelia and to the choice of a treatment method.

3. MRI is important for the postoperative follow-up of patients with syringomyelia, including tracking the cyst and detecting complications.

Keywords: Syringomyelia; magnetic resonance imaging; MRI.

\section{Introduction}

Since the advent of magnetic resonance imaging intramedullary abnormalities can be directly visualized. In this paper we described MRI findings in syringomyelia in general and the fluid motion in the syrinx cyst on phase shift imaging in particular. The clinical significance of this technique is demonstrated in a series of 15 patients.

\section{MRI in Syringomyelia}

Magnetic resonance imaging (MRI) is the most important neuroradiological method of investigation for suspected syringomyelia $[2,7,8]$. The advantages of MRI include [1, 2, 8-11]:

1. Demonstration of a syrinx cyst in a normal sized, or atrophic spinal cord, especially in cases of post-traumatic syringomyelia (Fig. 1).

2. Determination of the lower and upper borders of the cyst.
3. Determination of septa in the cysts (Fig. 2).

4. Detection of associated disorders, such as cysts in the posterior fossa (Fig. $11 \mathrm{a}$ and b), tethered cord, Chiari malformation etc. (Fig. 3).

5. Follow-up after treatment and detecting complications of treatment.

6. Study of fluid motion in the subarachnoid space and in the cyst using flow imaging techniques.

\section{Materials and Methods}

This article is derived from the results of MRI (and other) examinations in 19 patients, in whom the diagnosis of syringomye-

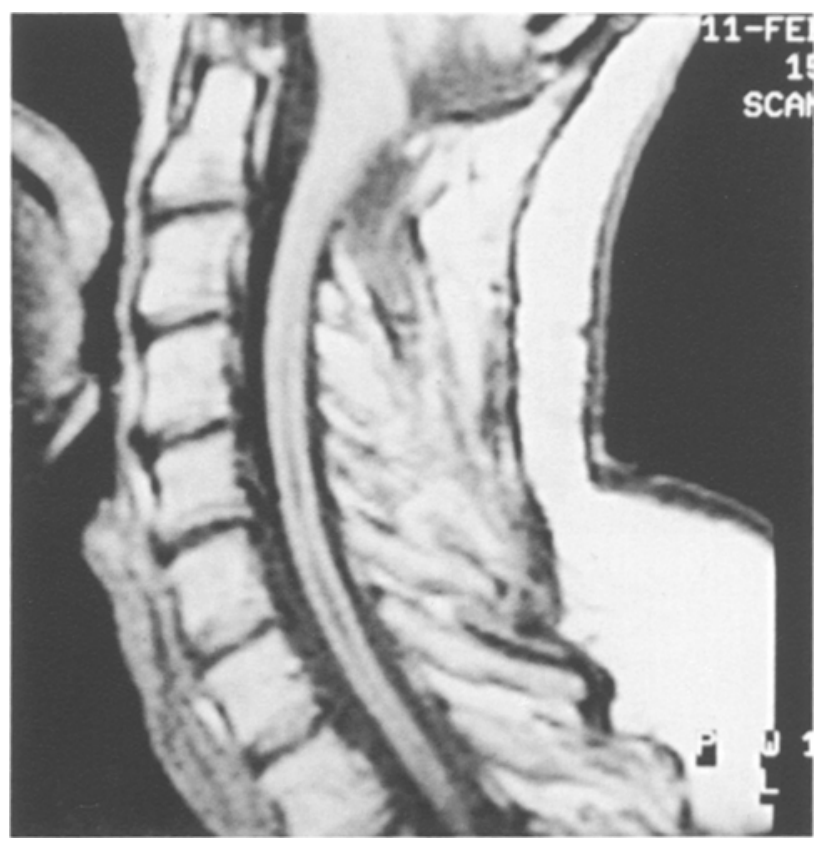

Fig. 1. Syrinx cyst in a spinal cord without enlargement (TIW image) 


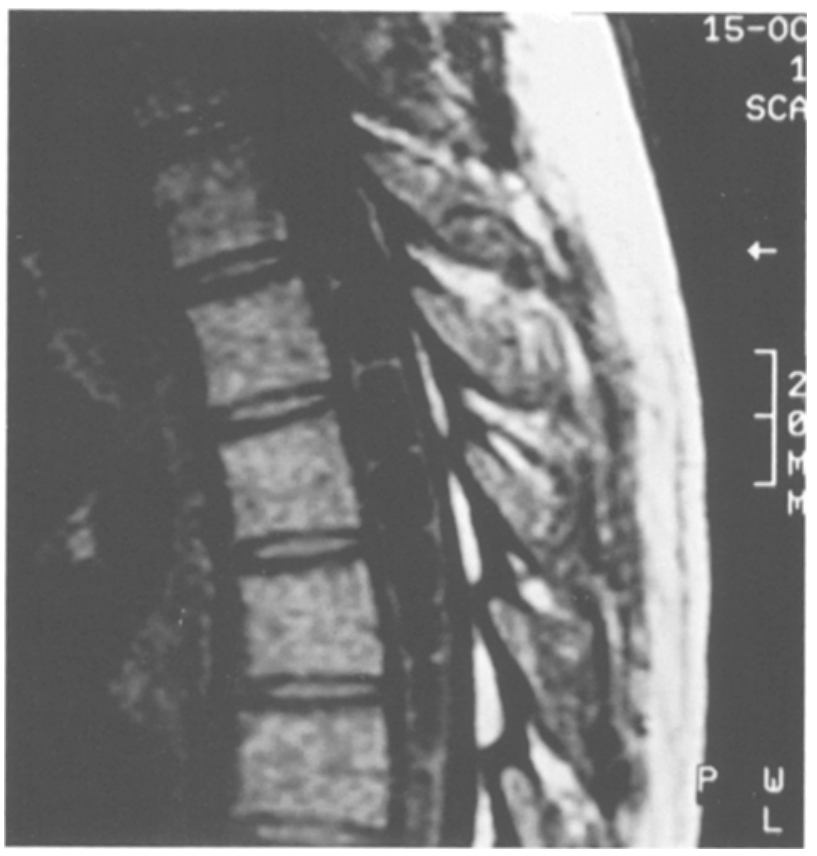

Fig. 2. Syrinx cyst with multiple septa (T1W image)

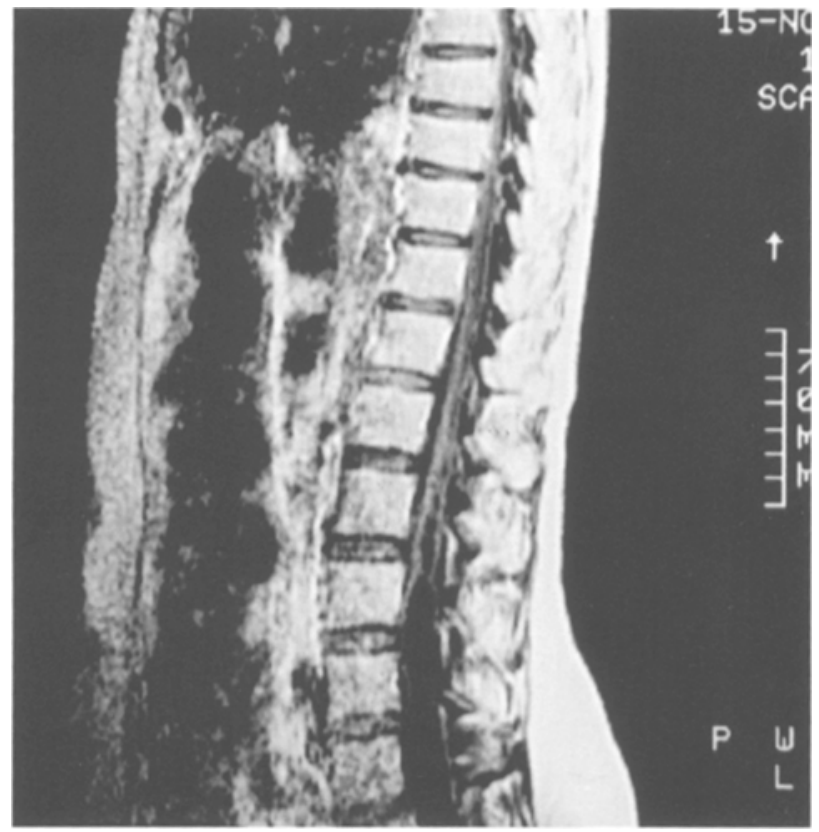

Fig. 3. Syringomyelia of the distal spinal cord in a patient with tethered cord. Also thickened filum terminale and sacral extradural arachnoid cyst (not shown) (T1W image)

lia was established on the basis of clinical neurological examination and previous neuroradiological investigations. The MRI examinations were performed according to a fixed protocol during the period December 1989 - September 1991

When assessing the results, the following parameters were con- sidered: the size of the cyst, the size of the spinal cord, septa in the cyst, the fluid motion on non-quantitative phase-shift imaging, the anatomical condition after foramen magnum decompression, the associated abnormalities, the evolution after treatment, and the position of a possible syringo-subarachnoid shunt.

\section{Results}

MRI failed to demonstrate a syrinx cyst in 4 of 19 patients. Two of these patients had no abnormalities, one had a Chiari malformation without a cyst, and one patient had an atrophic cervical spinal cord without cyst. Eleven of the 19 patients had an associated Chiari type I malformation and one patient had a Chiari type II malformation. There was one case of syringomyelia of the distal spinal cord in the context of a tethered cord with thickened filum terminale and sacral extradural arachnoid cyst. Two patients had a syringomyelia without associated abnormality. Patients with a post-traumatic syringomyelia have not been included in this study, although such cases were investigated during the period 1989-1991 [3].

\section{The Basic Features of Syringomyelia}

Syringomyelia has two basic features on MRI:

1. The presence of a cyst in the spinal cord parenchyma.

2. The associated abnormal signal of the spinal cord due to gliosis. This second feature was not further investigated in our protocol.

These features can be analysed in the T1-weighted image (T1W image) and in the T2-weighted image (T2W image).

The T1W image [8]: The sagittal T1W spin echo sequence is the basic sequence in every MRI investigation of the vertebral column and its contents. The
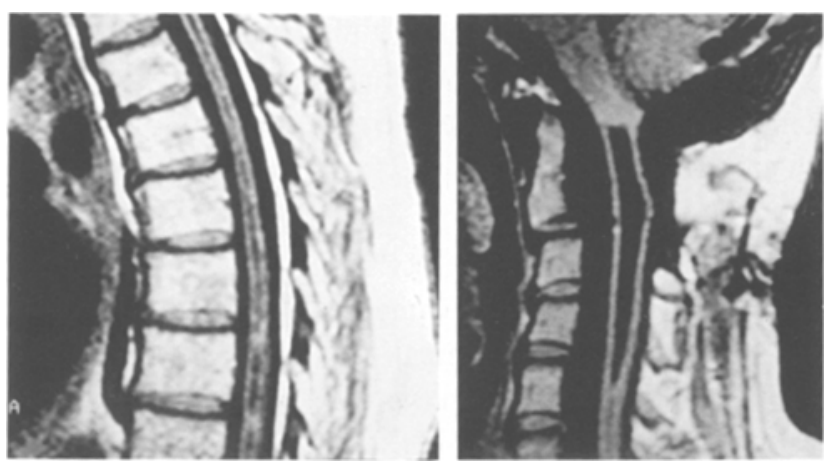

a

\section{b}

Fig. 4. (a) In a small non-pulsatile syrinx cyst the signal intensity is higher than the pulsatile CSF (T1W image). (b) In a large syrinx cyst the signal intensity is equal to CSF (T1W image). Status after foramen magnum decompression 


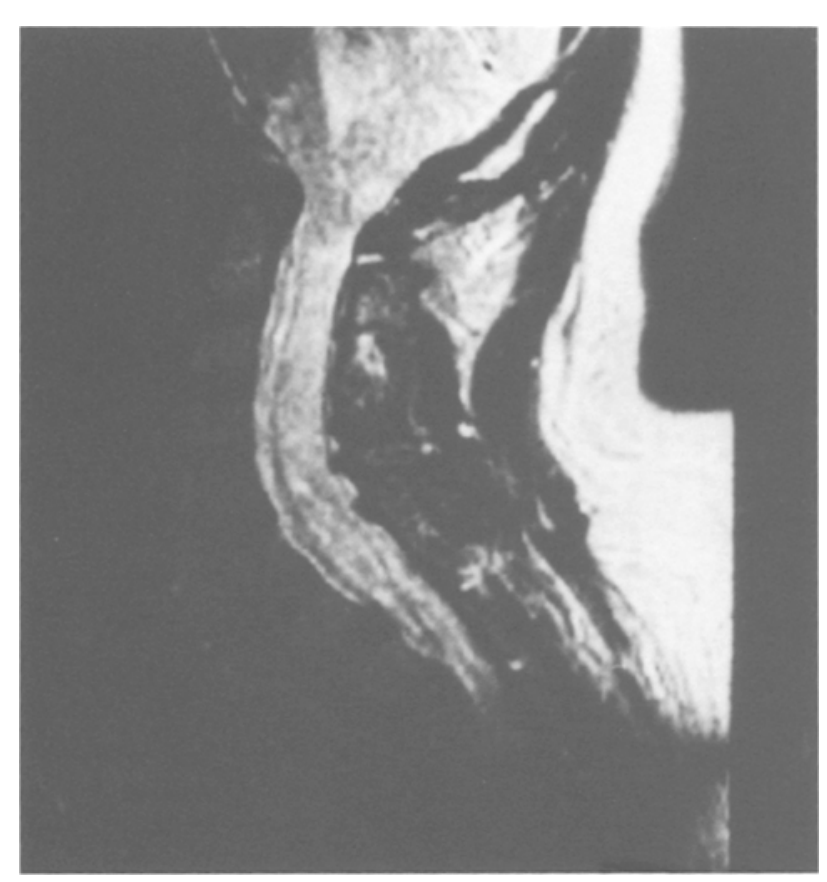

Fig. 5. Fluid motion in the syrinx cyst causes a hypo-intense signal (signal void) in the non-flow compensated $\mathrm{T} 2 \mathrm{~W}$ image

T1W image is particularly suitable for imaging normal anatomy and for disorders which are characterized by disturbed anatomy such as syringomyelia. The syrinx cyst has a signal intensity equal to or slightly higher than CSF, depending on the fluid motion. Small cysts with stationary fluid have a slightly higher signal intensity than the pulsating CSF. Larger cysts with pulsating fluid have a signal intensity similar to CSF (Fig. $4 \mathrm{a}$ and b). The size of the cysts is very variable and can range from a small focal cyst, as in post-traumatic syringomyelia, to a cyst which stretches from the cranio-cervical transition to the conus. The $\mathrm{T} 1 \mathrm{~W}$ image produces a more accurate reflection of cyst size than the T2W image, because it is less sensitive to flow effects. Septa in the cyst can best be seen in the T1W image.

The $T 2 \mathrm{~W}$ image [8]: The T2W image is particularly suitable for imaging pathological tissue characterized by an abnormal signal intensity and for investigating fluid dynamics. In a multi-echo sequence we obtain two images with a long TR: the proton density image (PDW image) with short TE and the "heavy T2W image" with long TE. Each has its own diagnostic value. In the non-flow compensated $\mathrm{T} 2 \mathrm{~W}$, pulsatile fluid gives a hypo-intense signal (signal void). This is also produced by fluid motion in the syrinx cyst (Fig. 5). In a syrinx cyst with several septa it is
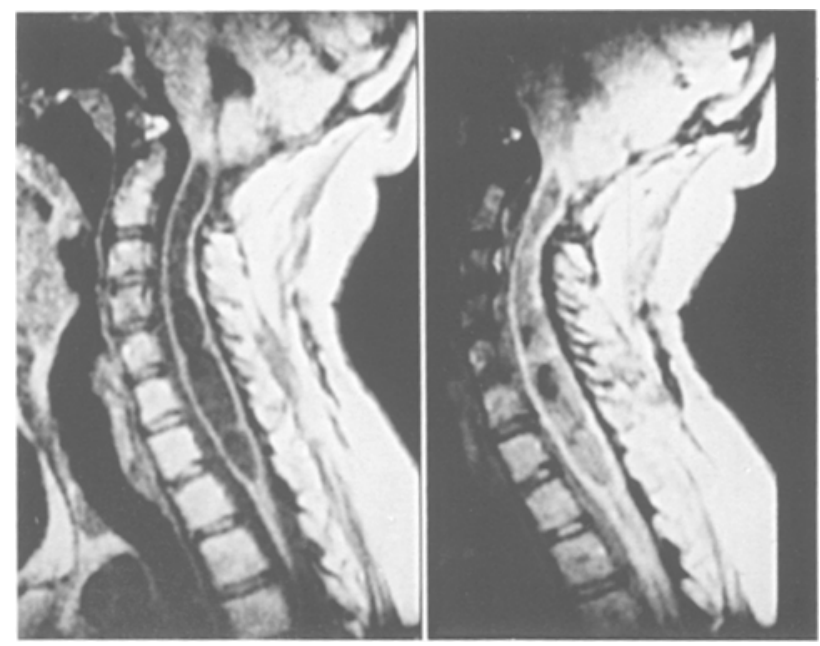

a

b

Fig. 6. Different signal intensities in the various compartments of a syrinx cyst with septa. (a) T1W image (left), (b) PDW image (right)

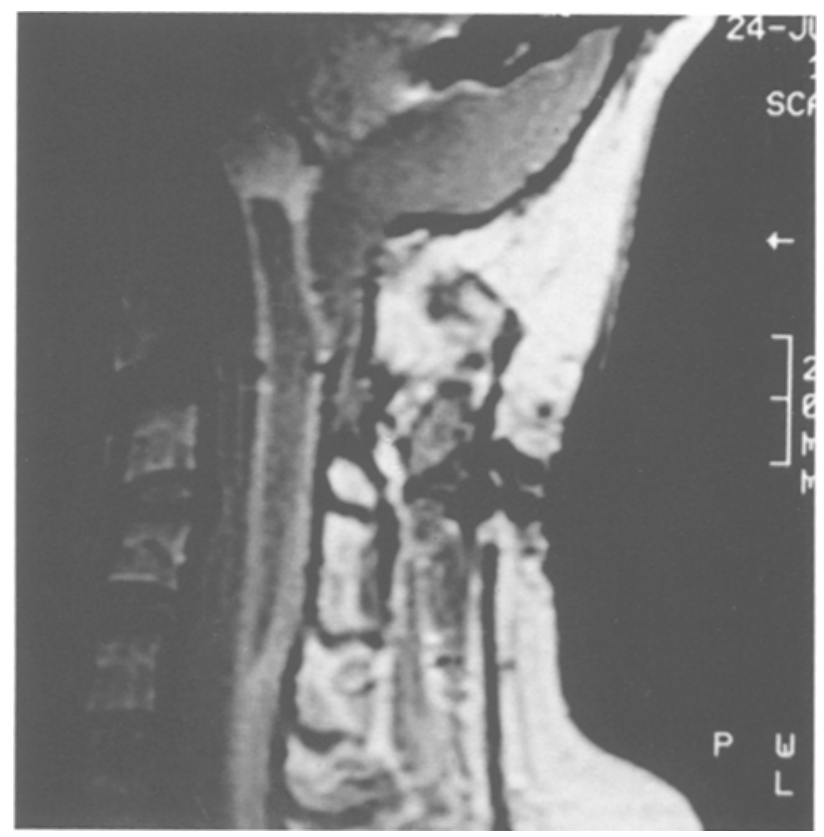

Fig. 7. Myelomalacia: abonormal signal of spinal cord tissue above the syrinx cyst

possible that the various compartments have different signal intensities due to differences in fluid motion (Fig. $6 \mathrm{a}$ and b). The spinal cord tissue around a syrinx cyst is often abnormal, giving a hyper-intense signal. We use a descriptive term for this tissue: myelomalacia (Fig. 7). Distinction between the static hyper-intense fluid of a syrinx cyst and myelomalacia 

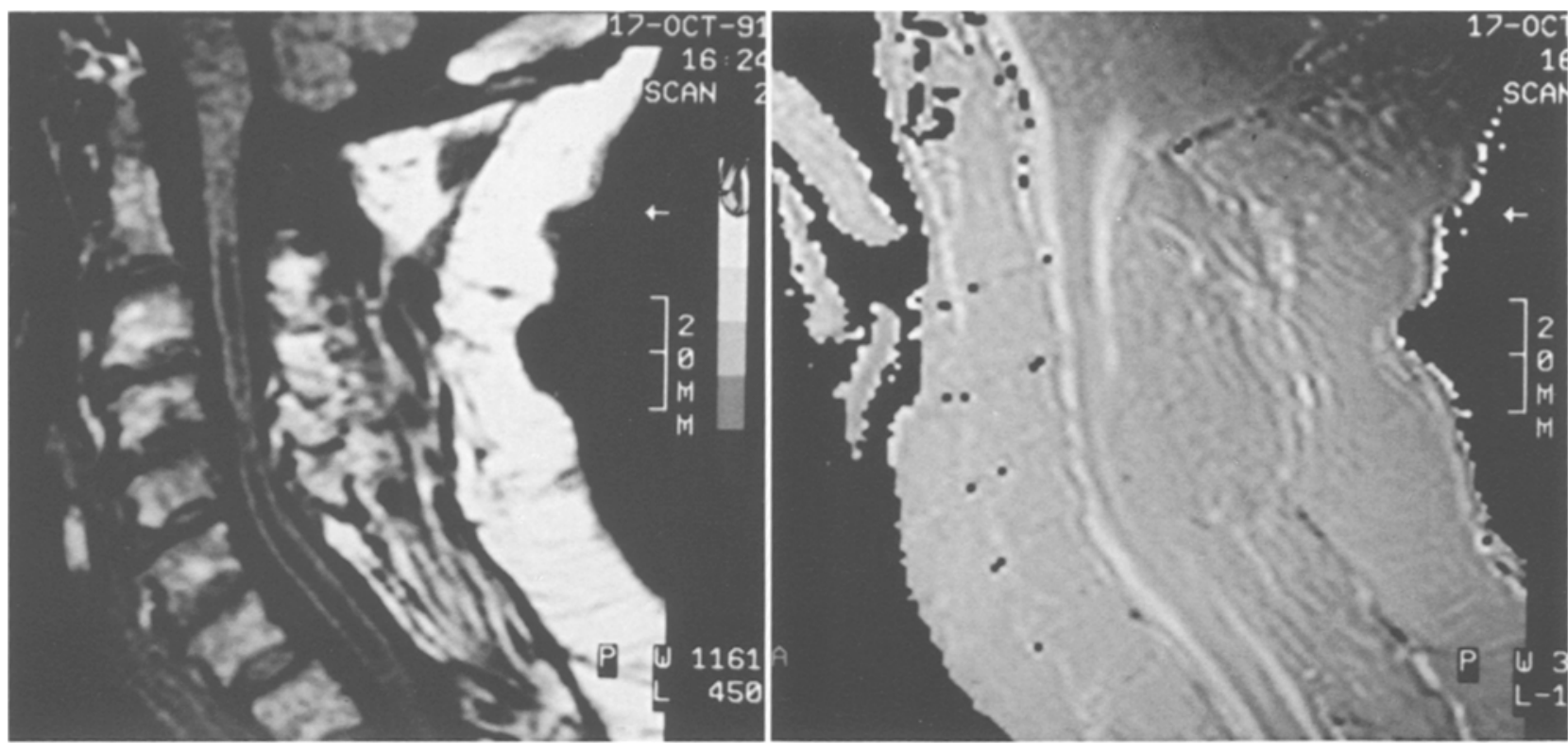

a

Fig. 8. (a) Equal signal intensity in syrinx cyst and adjacent subarachnoidal space (T1W image). (b) The pulsation in the syrinx is in close harmony with that in the adjacent subarachnoidal space (phase-shift)

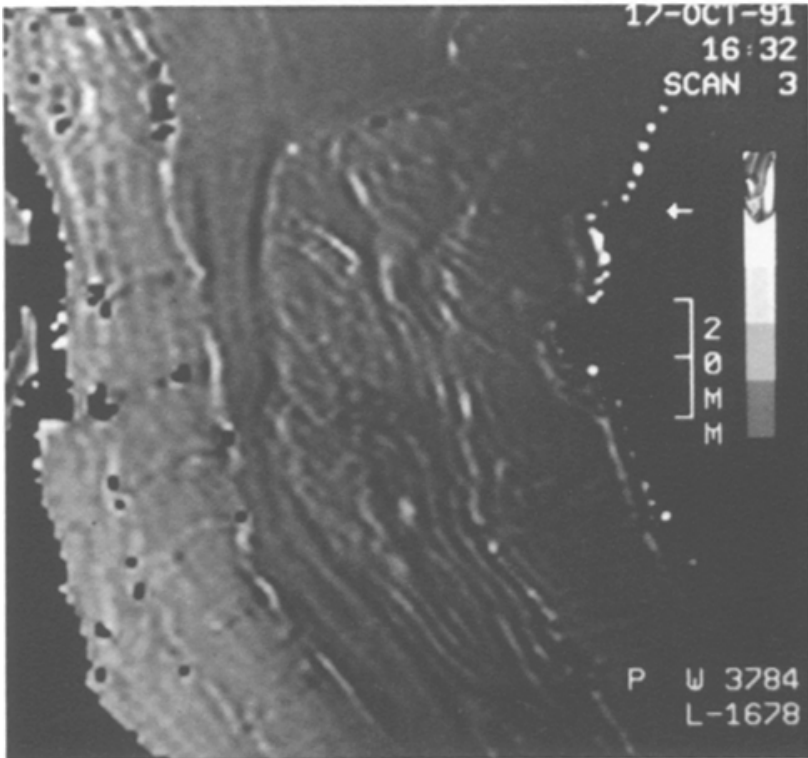

a

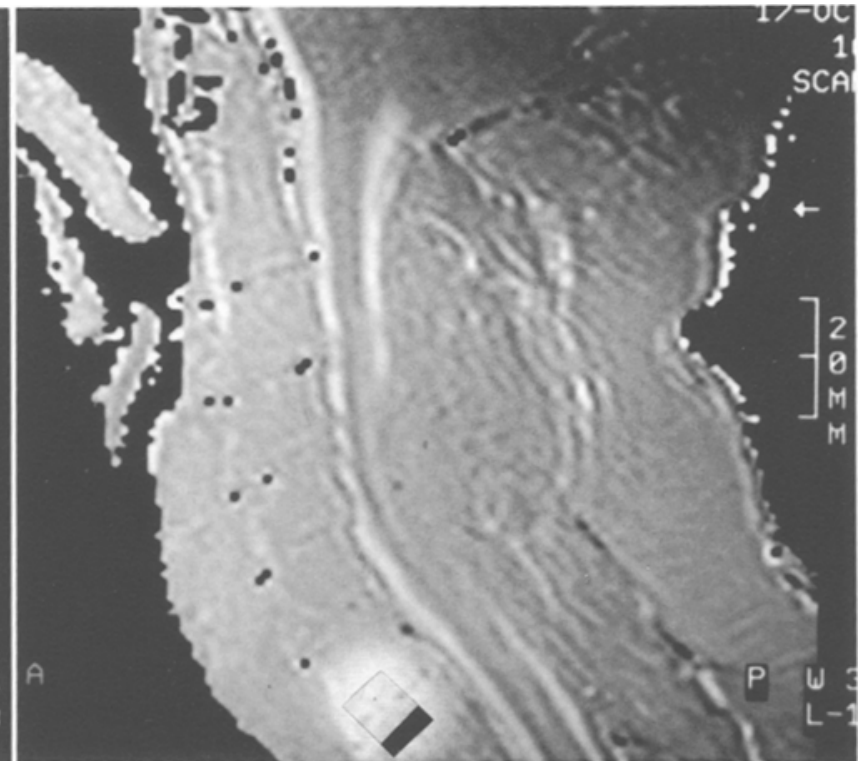

b

Fig. 9. (a) Phase-shift imaging corresponds with a cranio-caudal motion. (b) Phase-shift imaging during CSF diastole. White signal in the syrinx cyst corresponds with a caudo-cranial motion

may be difficult in the heavy $\mathrm{T} 2 \mathrm{~W}$ image. In the PDW image, myelomalacia is hyper-intense.

\section{The Study of Syringeal Fluid Motion}

In our series of 15 patients, the fluid motion in the syrinx cyst was investigated qualitatively using a non-flow compensated $\mathrm{T} 2$ spin echo sequence and by phase-shift imaging. The correlation with the clinical condition was investigated.

Studies on the pulsatile nature of the cervical CSF were performed in 1966 by Du Boulay. These observations were extended by Lane and Kricheff in 1972 
using video densitometry and lipiodol myelography. CSF-pulsations in the spinal canal were first described on MRI by Sherman in 1986.

In 1988, Rubin and Enzmann were the first to use an MR method for quantification of CSF flow. Quencer and Post described the technique of phasecontrast cine MR in 1988. MR methods [4, 9-11] for the qualitative study of fluid movement are non-flow compensated $\mathrm{T} 2 \mathrm{~W}$ image and phase-shift imaging. Quantitative MR methods are phase-contrast cine MR and dual flip angle technique $[5,8,9]$. The methods of non-motion-compensated $\mathrm{T} 2 \mathrm{~W}$ sequence have a threshold for the detection of fluid movement. This leads to a type of binary phenomenon: with movement/without movement. And so this method is really more qualitative than quantitative. It is only quantitative inasmuch as a particular pulse-amplitude threshold has to be exceeded.

The phase contrast cine MR technique, in a series of e.g. 16 images spread over the entire period of a heart beat cycle, produces dynamic information about the speed and direction of the fluid movement in the normal subarachnoid space and in the syrinx.

Two features of the fluid motion can be analysed: the speed (in $\mathrm{mm} / \mathrm{sec}$ ) and the direction. The coding of the direction is such that cranio-caudal flow is represented as a black signal, and caudo-cranial flow as a white signal. Absence of flow produces a grey signal [9-11].

The quantitative MR technique for studying fluid
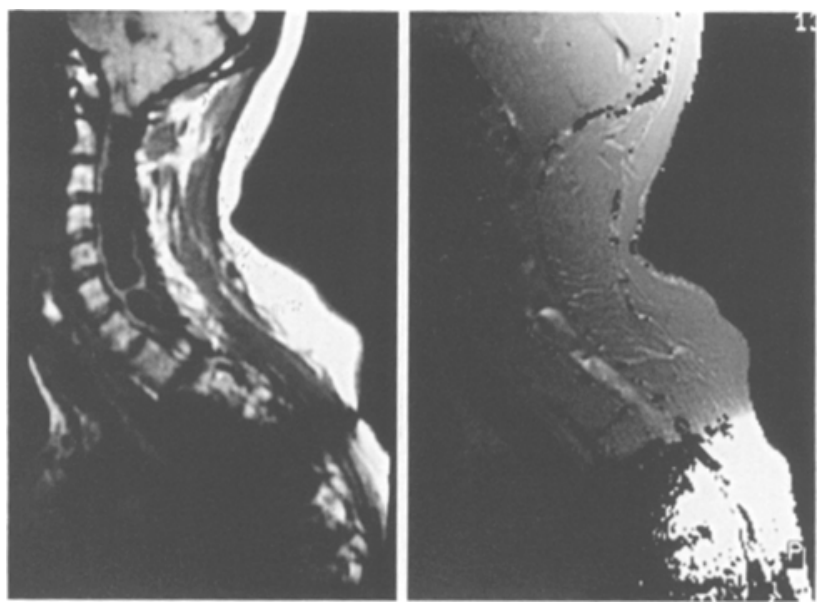

a

b

Fig. 10. $(a, b)$ Non-uniform fluid motion in a syrinx cyst with septa. Phase-shift imaging (b) shows different signal intensity in the various compartments of the syrinx cyst (a) motion is still in its infancy. It is not yet known how this information is correlated with the clinical symptomatology, the progressive of the disease or the response to treatment.

Just as little is known about whether there is a relationship between the severity of adjacent spinal column damage (myelomalacia) and the dynamics of the movement.

Data known so far:

1. The fluid motion in the syrinx cyst is pulsatile in nature.

2. The pulsation in the syrinx runs in close harmony with that in the adjacent subarachnoidal space (Fig. 8).

3. The fluid movement in the syrinx has a definite systolic and diastolic component, which run in parallel with those of the adjacent subarachnoidal space.

4. The systolic component is aimed cranio-caudally; it is seen in the last part of the heart beat cycle on the peripheral pulse metre recording, not on the ECG (Fig. 9 a).

5. The direction of the diastolic component is caudo-cranial and it lasts longer than the systolic component (Fig. 9 b).

6. Large syrinx cysts have a pulsatile wave which runs in systole from cervical to thoracic and the other way around in diastole. In general, this pulsatile wave
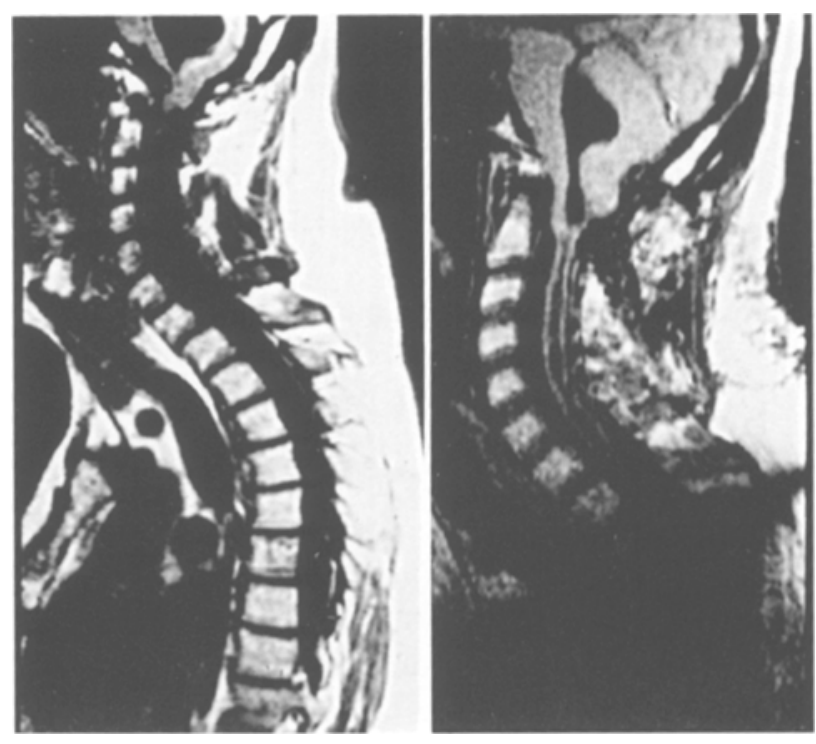

a

b

Fig. 11. (a) Syrinx cyst before treatment. Arnold-chiari type II malformation. (b) Diminution of the size of the cyst after treatment of coexisting hydrocephalus. Arnold-Chiari type II malformation 


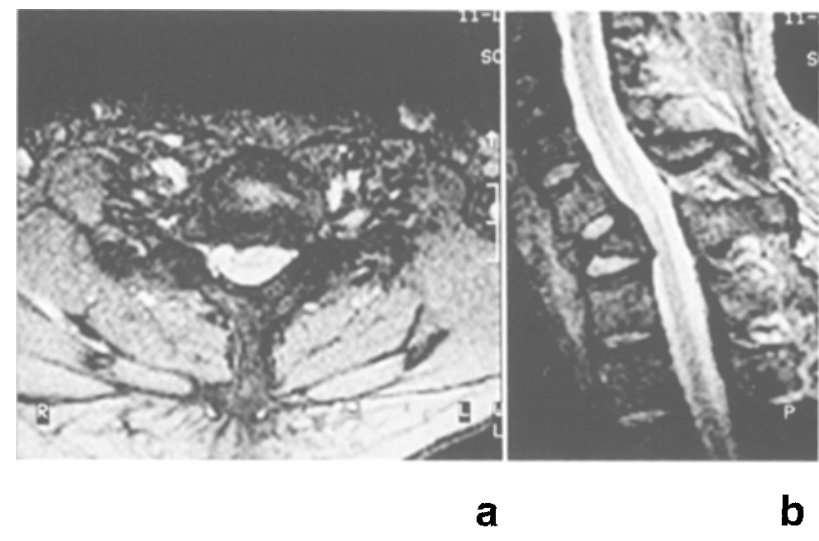

Fig. 12. Myelomalacia in a patient with paraplegia after a cervical trauma with a teardrop fracture. (a) Hyperintense intramedullary lesion on $\mathrm{T} 2 \mathrm{~W}$ image in the axial and (b) sagittal plane

runs synchronously with the pulsatile wave of the surrounding subarachnoidal space.

7. The fluid movement in the syrinx cyst is relatively uniform, but turbulence can occur near septa (Fig. 10.).

8. Postoperatively, the fluid movement often decreases in proportion to the reduction in size of the cyst. In a cyst which has almost collapsed, the fluid becomes stationary [8].

\section{MRI in Postoperative Syringomyelia}

In our series, MRI was carried out in ten patients in the postoperative phase, in two patients both pre- and postoperatively and in three patients only pre-operatively. We looked at:

1. The anatomical condition of the cranio-cervical transition after foramen magnum decompression, and possible complications;

2. The cyst size and fluid movement;

3. The position of a possible shunt catheter. Usually, it was impossible to image the shunt catheter because of local metal artefacts.

The MRI criterion for a "successful" treatment is the diminution of the cyst (Fig. 11 b). It is not clear whether the syringeal fluid motion also has to decrease. In a series of 20 postoperative patients of Barkovich [2], a signal void remained in the cyst in ten patients, without clinical deterioration at a followup of one year (possibly too short). But in all seven patients in whom the cyst was diminished, the syringeal fluid motion also decreased and the number of patients with signal void in the cyst was larger preoperatively (11/12 patients) [2].

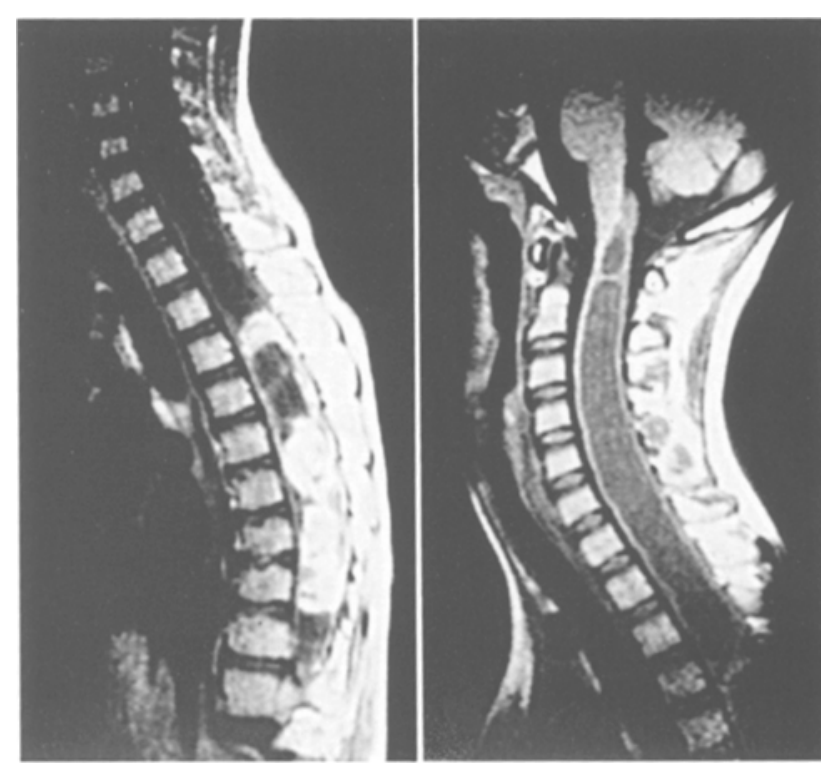

a

b

Fig. 13. Spinal cord tumour with cyst. (a) Gadolinium enhanced T1W image of the tumour (left). (b) Fluid in tumour cyst has a higher signal intensity due to higher protein concentration (right)

\section{Differential Diagnosis}

The truncation artefact: The "overdiagnosis of syringomyelia" stemming from the first days of MRI, can be blamed on confusion with the truncation artefact. This artefact is a direct consequence of using the Fourier transformation to create an image. In the cervical spinal cord, this can produce a hypo-intense line in the centre. This band is, however, never as hypointense as the signal produced by CSF [6].

Myelomalacia: Late progression of the neurological sequelae following spinal trauma can be due to causes other than syringomyelia. For example: hypertrophy of the posterior longitudinal ligament, posttraumatic spondylosis deformans with osteophyte formation, traumatic disc prolapse, arachnoiditis, "tethering" of the signal cord, post-traumatic intradural arachnoidal cyst with spinal compression and myelomalacia. They are all visible on MRI. Myelomalacia (softening of the spinal cord) is a descriptive term. The neuropathological substrate is a combinatin of neuronal loss, demyelinization, infarct and gliosis. There may be accompanying microsysts [7]. On MRI, these can be confused with a syrinx cyst: hypo-intense on the T1W image, hyper-intense on the T2W image. But a zone of myelomalacia is hyper-intense on the PDW image, while the syrinx cyst looks the same as CSF [7] (Fig. 12 a, b). 
Spinal cord tumour with cyst (Fig. 13 a, b): On the T1W image the differential diagnosis between syringomyelia and a large tumour cyst can be difficult. Zones of spinal cord swelling without cyst are indicative of a tumour. The fluid in a tumour cyst often has a higher signal intensity due to higher protein concentration amongst other things. On the T2W image, both a tumour cyst and syrinx cyst are hyper-intense. Inhomogeneous signal intensity (e.g. due to previous bleeding) and a hyper-intense signal outside the cystic zone, because of spinal cord edema or tumour tissue (mainly on the T1W image), indicate a tumour. The MR-contrast agent, Gadolinium = DTPA, facilitates differentiation. A syrinx cyst does not stain while, according to current experience, all spinal tumours do stain. Accompanying abnormalities of the cranio-cervical transition and a cyst in occult spinal dysraphism make a spinal tumour highly improbable.

\section{References}

1. Banna M (1989) Syringomyelia in associatin with posterior fossa cyst. AJNR 9: 867-873

2. Barkovich AJ, Sherman JL, Citrin, et al (1987) MR of postoperative syringomyelia. 8: 319-327

3. Batzdorf U (1991) Classification of syringomyelia. In: Batzdorf U (ed) Syringomyelia. William and Wilkins, Baltimore
4. Bradley WG, Nitz W (1991) CSF velocity imaging in the spine. In: Hasso, Stark (eds) Categorical course syllabuss: spine and body MRI of American Röntgen Ray Society, 90th Annual Meeting, 5-10 May, 1991

5. Castillo M, Quencer RM, Green BA, et al (1987) Syringomyelia as a consequence of compressive extramedulary lesions: postoperative, clinical and radiological manifestations. ANJR 8: 973-978

6. Czervionke LF, Czervionke JM, Daniels, et al (1988) Characteristic features of MR truncation artefacts. AJNR 9: 815-824

7. Enzmann DR (1990) Syringomyelia. In: Enzmann DR, De La Paz RL, Rubin JB (eds) Magnetic resonance of the spine. Mosby, St Louis

8. Enzmann DR (1991) Imaging of syringomyelia. In: Batzdorf U (ed) Syringomyelia. Williams and Wilkins, Baltimore

9. Enzmann DR, Pelc M (1991) Normal flow patterns of intracranial and spinal cerebrospinal fluid defined with phase-contrast cine MR imaging. Radiology 178: 467-474

10. Levy LM, DI Chiro G (1990) MR phase imaging and cerebrospinal fluid flow in the head and spine. Neuroradiology 5: 399-406

11. Quencer RM, Donovan Post MJ, Hinks RJ, et al (1990) Cine MR in the evaluation of normal and abnormal CSF flow: intracranial and intraspinal studies. Neuroradiology 5: 371-391

12. Wiliams B (1991) Pathogenesis of syringomyelia. In; Batzdorf $\mathrm{U}$ (ed) Syringomyelia. Williams and Wilkins, Baltimore

Correspondence: H. L. J. Tanghe, M.D., Department of Radiodiagnostics, University Hospital Dijkzigt, Dr. Molewaterplein 40, 3015 GD Rotterdam, The Netherlands. 